\title{
ORIGINAL RESEARCH \\ ACTIVATION OF VASTUS MEDIALIS OBLIQUES AT DIFFERENT KNEE ANGLES IN CLOSED KINETIC CHAIN AND OPEN KINETIC CHAIN POSITION IN SUBJECTS WITH PATELLO FEMORAL PAIN SYNDROME
}

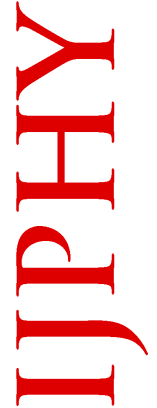

\author{
${ }^{1}$ Ujwal Bhattacharya \\ ${ }^{2}$ R. Sreekar Kumar Reddy
}

\section{ABSTRACT}

Background: Patello femoral pain syndrome is one of the most common musculoskeletal disorders and is reported to affect $15 \%-33 \%$ of an active adult population and $21 \%-45 \%$ of adolescents thereby decreasing the work capacity of an individual. Patello femoral pain syndrome as implicated is due to inappropriate neural control of the quadriceps femoris muscle. Since Vastus medialis muscle is regarded as the dynamic medial stabilizer of patella so it's important to understand the activation of Vastus Medialis Oblique's in patients with patello femoral pain syndrome The purpose of this study is to assess the activation of Vastus Medialis Oblique's at different knee angles between closed kinetic chain position and open kinetic chain position and the information thus gained can be used to design training program aimed at controlling patello femoral joint dysfunction.

Methods: Thirty subjects with patello femoral pain syndrome (assessed through history and examination) were assigned to two groups. Group A was made to perform open kinetic chain activity(i.e. sitting on high plinth) and Group B was made to perform a closed kinetic chain activity(i.e. standing) The outcome measure were amplitude and duration. Analyses were performed using independent t-Test.

Results: Significant difference was found between the groups for amplitude and duration at varying angles. Amplitude: 0 degree $(p=0.004), 60$ degrees $(p=0.015), 90$ degrees $(p=0.004)$. Duration: 0 degree $(p=0.007), 60$ degrees $(p=0.008), 90$ degrees $(p=0.002)$

Conclusion: Based on the outcome of the study it's concluded that there is greater activation of Vastus Medialis Oblique's muscle while performing close kinetic chain activity than in open kinetic chain.

Key words: PFPS - Patello femoral pain syndrome, CKC - Close kinetic chain position,OKC - Open kinetic chain position, AKP- anterior knee pain.

Received $31^{\text {st }}$ March 2015, revised $08^{\text {th }}$ May 2015, accepted 26 ${ }^{\text {th }}$ May 2015

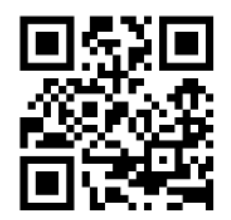

DOI: 10.15621/ijphy/2015/v2i3/67020

www.ijphy.org

\section{CORRESPONDING AUTHOR}

\section{${ }^{1}$ Ujwal Bhattacharya}

\footnotetext{
${ }^{2}$ Professor,

Narayana College of physiotherapy,

Nellore, Andhrapradesh.
}

Associate Professor,

Department of Physiotherapy,

College of physiotherapy and medical

sciences, Bamunimaidam,

Guwahati, Assam-781021 


\section{INTRODUCTION}

Patello femoral pain syndrome (PFPS) is one of the most common musculoskeletal disorder ${ }^{1}$ and is reported to affect $15 \%-33 \%$ of active population and $21 \%-45 \%$ of the adolescents. Among adolescents the incidence reported to be higher for girls. $^{2}$

PFPS effects athletes and non-athletes of both gender and is consistently reported in activity such as ascending or descending stairs and squatting or subsequent long periods of sitting still this regarded as the most important information in patients history. ${ }^{3}$

Patients commonly report that the development of their pain is insidious and that the pain is either sharp and acute or diffuse and chronic. PFPS sometimes begins when loading of the patello femoral joint suddenly increases. Military recruits of both genders are prone to develop PFPS. The true cause of PFPS is unknown and the pain syndrome has been given various names.

Anterior knee pain - A symptom common in traumatic and overuse knee disorder is often used synonymously with PFPS. ${ }^{4}$ Anterior knee pain is often diagnosed as chondromalacia patella but is only correct when softened and fissured patella under surface is seen during diagnostic imaging. ${ }^{5}$

Lieb and Perry separated Vastus Medialis muscle in two functional components based on fiber orientation with proximal longitudinal being termed Vastus Medialis Longus muscle and the distal oblique fibers being termed the Vastus Medialis Oblique muscle.As a result of its more horizontal fiber orientation Vastus Medialis Oblique (VMO) is considered primary medial stabilizer of patella. ${ }^{6}$

Vastus Medialis muscle is the dynamic medial stabilizer of patella therefore it is important to understand the function of VMO while treating patients with PFPS. ${ }^{7}$

Despite the large emphasis on the VMO in the treatment of PFPS assessment of VMO force production is not possible.In lieu of this limitation EMG has been used to establish the activity pattern of VMO on patella tracking in subjects with PFPS.

Although the cause of PFPS is obscure the most widely accepted hypothesis of pathology of PFPS is related to Patellar maltracking with Patellofemoral joint stress which progresses to articular cartilage degeneration. ${ }^{8}$ Reports however, have questioned a causal relationship between malalignment and knee discomfort in majority of PFPS patient ${ }^{9}$ and the phenomenon of rest pain has never been satisfactorily explained. A different etiology that takes a pain mechanism into consideration proposes that the etiology of PFP arises from a loss of homeostasis in PF tissues.

Ischemia, caused by high interroseous pressure has been proposed as a plausible pain mechanism. ${ }^{10}$ Studies by Bjorkstrom and Slater on cadaveric patella found articular cartilage degeneration concomitant with disrupted blood supply. ${ }^{11}$ Using interroseous venography Waisbrod observed that articular cartilage degeneration was accompanied by decreased venous out flow.

Arnoldi found higher intraosseous pressure in both the femur and the tibia in painful knees compared with healthy knees. He found a link between knee pain and intraosseous patellar pressure. He also demonstrated that painful knees often have limited venous outflow. ${ }^{12}$

\section{METHODOLOGY}

30 subjects both males and females with age group of 15-35 years were included with fulfillment of inclusion criteria, subjects with Any history of inflammatory disorders to the knee, Previous history of soft tissue injury to the knee, Any history of fracture to the knee, Any history of infective conditions around the knee, Any degenerative disorders around the knee joint were excluded.

\section{Interventions}

The study included 30 patients taken in a randomized sampling manner who have been diagnosed as having patellofemoral pain syndrome. Both males and females were taken into consideration. Before undergoing the intervention consent of the subjects is taken and is being properly assessed according to the assessment format given in the appendix.

\section{Group - A}

Open kinetic chain position (i.e., sitting on a high plinth)

Each subject was made to perform the open kinetic chain activity in which the subjects was made to sit on a high plinth and then EMG readings were documented with the knee in full extension (i.e, $0^{0}$ ). The subjects was instructed to flex the knee in the same position to varying degrees (i.e., $60^{\circ}$ and $90^{\circ}$ ) which were measured with Goniometer and in these positions EMG readings were documented again.

\section{Group - B}

Close kinetic chain position (i.e., standing)

The same subjects were made to perform the close kinetic chain activity in which the subjects was made to stand with the knee in full extension and the EMG readings were documented. 
The subjects was instructed to flex the knee in the same position to varying degrees (i.e., $60^{\circ}$ and $90^{\circ}$ ) which were measured with Goniometer and in these positions EMG readings were documented again.

Figure 1: Electrode placement

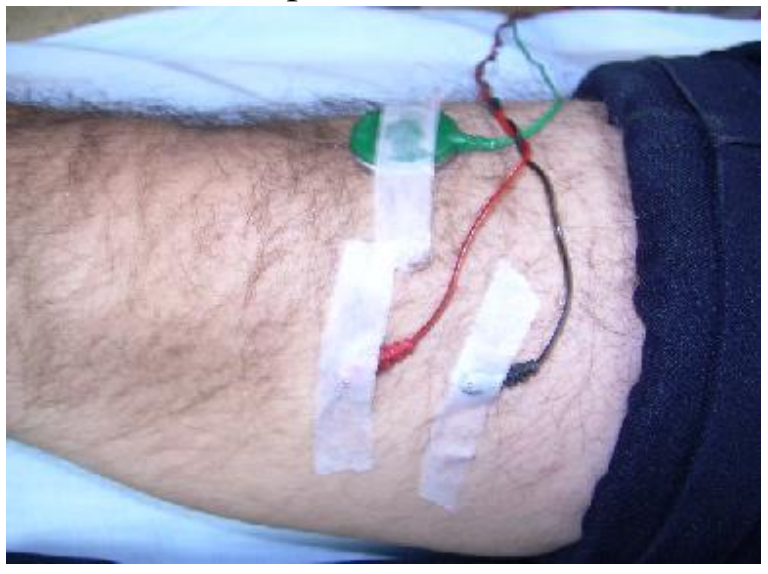

\section{RESULTS}

STATISTICAL ANALYSIS: The data achieved from the EMG findings of the OKC position and CKC position at varying angles are finally analyzed by determining the mean value ( $p$ value ) for gender distribution, amplitude of contraction and duration of contraction. The findings are plotted in graphical representation as follows:

Table 1: Summarizes the descriptive statistics of the gender distribution

\begin{tabular}{|c|c|c|c|c|} 
& Males & Females & $\mathrm{X}^{2}$ value & $\mathrm{p}$-value \\
\hline Group & 17 & 13 & 0.533 & $>0.465$ \\
\hline
\end{tabular}

Included for the study showing a significant mean value. ( $\mathrm{p}->0.465)$

Graph 1: Represents the gender distribution
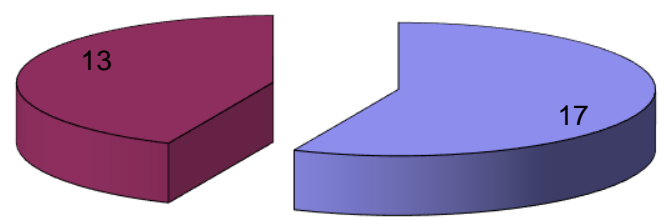

$$
\text { 口Males } \quad \text { Females }
$$

Table 2: Mean Values of Amplitude

\begin{tabular}{|c|c|c|c|}
\hline DEGREE/ & OKC & CKC & p \\
\hline ROM & VALUE \\
\hline 0 degree & $228.13 \pm$ & $240.03 \pm$ & .004 \\
\hline \multirow{2}{*}{ 60 degree } & $248.46 \pm$ & 14.3 & $.004 \pm$ \\
& 19.2 & 21.6 & .015 \\
\hline 90 degree & $290.23 \pm$ & $309.87 \pm$ & .004 \\
& 15.5 & 32.11 & .015 \\
\hline
\end{tabular}

Graph 2: Represents the mean values of the amplitude attained during activation of VMO in CKC as well OKC position at varying angles $\left[0^{\circ}(\mathrm{p}-\right.$ $\left.0.004), 60^{\circ}(\mathrm{p}-0.015), 90^{\circ}(\mathrm{p}-0.004)\right]$.It can be seen from the plotted graph that VMO activates better during CKC position.

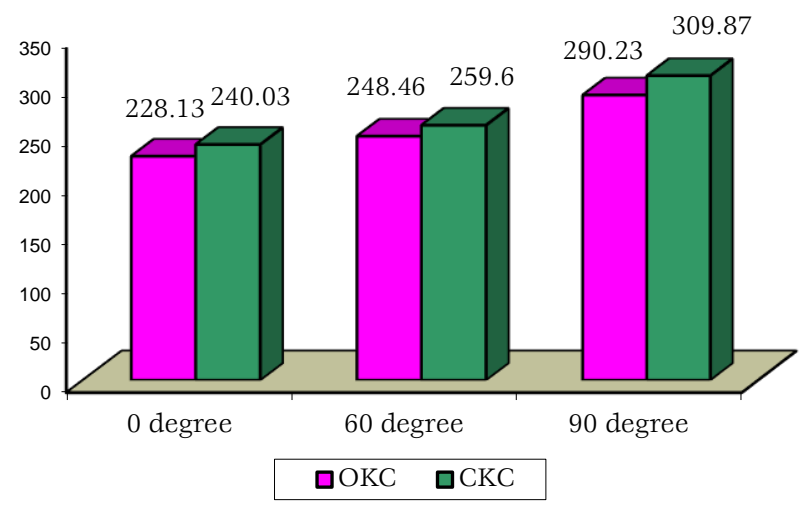

Table 3: Mean values of duration

\begin{tabular}{|c|c|c|c|}
\hline $\begin{array}{c}\text { DEGREE/ } \\
\text { ROM }\end{array}$ & OKC & \multicolumn{1}{c}{ CKC } & P VALUE \\
\hline 0 degree & $.007 \pm .002$ & $.005 \pm .001$ & .007 \\
\hline 60 degree & $.005 \pm .001$ & $.006 \pm .002$ & .008 \\
\hline 90 degree & $.004 \pm .001$ & $.003 \pm .001$ & .002 \\
\hline
\end{tabular}

Graph 3: Represents the mean values of the duration attained during activation of VMO in CKC as well OKC position at varying angles $\left[0^{\circ}(\mathrm{p}-\right.$ $\left.0.007), 60^{\circ}(p-0.008), 90^{\circ}(p-0.002)\right] . I t$ can be seen from the plotted graph that VMO activates better during CKC position showing more better fiber recruitment.

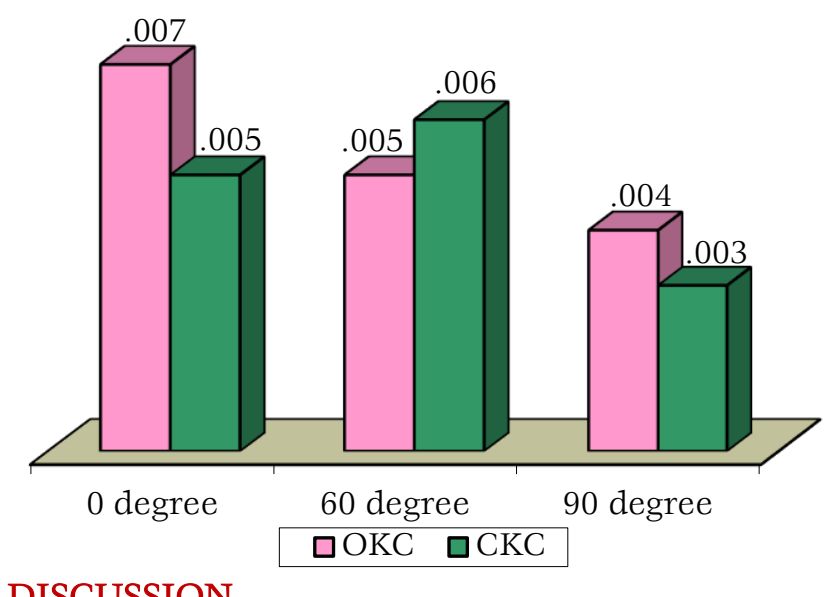

This study was intended to compare the activation of VMO at different knee angles between close kinetic chain position and open kinetic chain position in subjects with patellofemoral pain syndrome. This study showed significant difference in levels of activation of VMO at three specific knee flexion angles (i.e., $0^{\circ}, 60^{\circ}$ and $90^{\circ}$ ) between close kinetic chain and open kinetic chain 
activities with regards to amplitude and duration values in subjects with PFPS.

This study shows the mean values of the amplitude attained during activation of VMO in CKC as well OKC position at varying angles $\left[0^{\circ}(p-\right.$ $\left.0.004), 60^{\circ}(\mathrm{p}-0.015), 90^{\circ}(\mathrm{p}-0.004)\right]$.

This study represents the mean values of the duration attained during activation of VMO in CKC as well OKC position at varying angles $\left[0^{\circ}(\mathrm{p}-\right.$ $\left.0.007), 60^{\circ}(p-0.008), 90^{\circ}(p-0.002)\right]$. The $p$-value is less than 0.05 in mean amplitude during activation of VMO.

Thus this study suggests that VMO activation was comparatively more in close kinetic chain activity than in open kinetic chain activity in all the 3 specific knee flexion angles (i.e., $0^{\circ}, 60^{\circ}$ and $90^{\circ}$ ) in subjects with PFPS.

The results of this study are in accordance with the previous study done by Lam PL, Ng GY, (2001). In his study on activation of quadriceps muscle during semi squatting with different hip and knee positions in patients with anterior knee pain concluded that there was relatively more activation of VMO than VL at $40^{\circ}$ of semi squat with the hip medially rotated by $30^{\circ}$.

Thus these finding has clinical implication for training VMO in patients with PFPS.

Since Patello femoral pain syndrome is one of the most common cause for anterior knee pain it is very necessary to find out the activation of Vastus medialis obliques muscle in various activities involving closed and open kinetic chain positions. Thus this study has been carried out with an aim to analyze the levels of activation of VMO in CKC as well as OKC positions at different knee angles by means of EMG analysis.

The results of the study revealed that in CKC position the activation of VMO was greater than in OKC position .Even among the three knee angles (i.e., $0^{\circ}, 60^{\circ}, 90^{\circ}$ ) in CKC position the activation of VMO was much greater in 90 degrees than in other angles .Although there was activation of VMO in OKC position but the activation level was less efficient compared to CKC position.

Thus it is concluded from the study that VMO activation was much greater in CKC than in OKC position, so it is recommended that the findings of this study can be clinically implicated in the rehabilitation programme for training VMO in patients diagnosed with Patello femoral pain syndrome.

\section{CONCLUSION}

Based on the outcome of statistical analysis it can be seen that there is greater activation of vastus medialis muscles in closed kinetic chain activity. Thus we can conclude that vastus medialis obliques muscle is more active during closed kinetic chain activity than in open kinetic chain activity.

\section{REFERENCES}

1. Murray IR, Murray AS, Mekenzie K, Coleman S. How evidence based is the management of two common sports injuries in a sports clinic?. Br J Sports Med.2005; 39:912-916.

2. Lindberg U. The patelofemoral pain syndrome, Thesis, Linkoping University, Linkoping Sweden, 1986.

3. Kristin M Houghton. Review for the generalist: evaluation of anterior knee pain. Pediatr Rheumatol Online J. 2007; 5: 8.

4. Van Tiggelen D, Witvrouw E, Roget P, Cambier D, Danneels L, Verdon k R. Effect of bracing on the prevention of anterior knee pain - a prospective randomized study. Knee Surg Sports Traumatol Arthro Sc. 2004;12(5):434-9.

5. Fulkerson, JP and Hungerford DS. Disorders of patello femoral joint. $2^{\text {nd }}$ edition; 1990.

6. Lieb $\mathrm{R}$ and Perry J.Quadriceps functions. JBJS.1968;50A(8), 1535-1548.

7. Peter L. Williams. Grays Anatomy.38 ${ }^{\text {th }}$ edition;1995.

8. David C Reid.Sports injury and assessment and rehabilitation. $1^{\text {st }}$ edition; 1991.

9. Dye SF, The pathophysiology of patello femoral pain. Clinc orthop Rel Res. 2005; 436:100-10.

10. Janice K. Loudo, Doug Wiesner. Intrarater Reliability of Functional Performance Tests for Subjects with Patellofemoral Pain Syndrome. J Ath1 Train. 2002;37(3): 256-261.

11. Warmslay R. The development of Patella. J Anat. 1940;74(Pt 3): 360-368.3.

12. Bischoff JE1, Hertzler JS, Mason JJ. Patellofemoral interactions in walking, stair ascent, and stair descent using a virtual patella model. J Biomech. 2009;42(11):1678-84.

\section{Citation}

Bhattacharya, U., \& Reddy, S. (2015). ACTIVATION OF VASTUS MEDIALIS OBLIQUES AT DIFFERENT KNEE ANGLES IN CLOSED KINETIC CHAIN AND OPEN KINETIC CHAIN POSITION IN SUBJECTS WITH PATELLO FEMORAL PAIN SYNDROME. International Journal of Physiotherapy, 2(3), 487-490. 